\title{
Fetal uvula: navigating and lightening the soft palate using HDlive
}

\author{
Gabriele Tonni • Gianpaolo Grisolia
}

Received: 5 April 2013/Accepted: 4 May 2013/Published online: 21 May 2013

(C) Springer-Verlag Berlin Heidelberg 2013

\section{Introduction}

Cleft lip and palate (CLP) have an incidence of 1.82 in 1,000 live births [1], thus representing one of the most common congenital anomalies. In a series by Cash et al. [2], $67 \%$ of the cases involved isolated cleft lip, $93 \%$ involved CLP, and only $22 \%$ involved isolated cleft palate. The prenatal detection of 553 cleft lips with or without cleft palate and 198 cleft palates by 2D ultrasound from 20 European registries was reported by Clementi et al. [3]. Prenatal diagnosis of CLP was made in 65/366 cases with an isolated malformation, 32/62 cases with chromosomal anomaly, 30/89 cases with multiple malformations, and 21/36 syndromic cases. Prenatal diagnosis of isolated cleft palate was made in only $13 / 198$ cases. Prenatal ultrasound diagnosis of CLP in a low-risk population has proved to have a low detection rate that ranged from 9 to $100 \%$ for cleft lip with or without cleft palate, 0-22\% for isolated cleft palate only, and 0-73\% for all types of cleft. 3Dultrasound in high-risk women (women whose fetuses were suspected or confirmed to have facial cleft by 2D ultrasound examination or who had a family history of facial cleft) yielded a detection rate of $100 \%$ for cleft lip, 86-90 \% for cleft lip with palate, and 0-88.9\% for cleft primary palate only [4]. In a recent series, Maarse et al. [5] studied 35,924 low-risk and 2,836 high-risk pregnant

\footnotetext{
G. Tonni $(\bowtie)$

Department of Obstetrics and Gynecology, Prenatal Diagnostic Center, Guastalla Civil Hospital, ASL Reggio Emilia,

Via Donatori di Sangue, 1, 42016 Guastalla, RE, Italy

e-mail: tonni.gabriele@ausl.re.it; tonnig@ausl.re.it

G. Grisolia

Department of Obstetrics and Gynecology, Prenatal Diagnostic Center, "Carlo Poma" Hospital, Mantua, Italy
}

women undergoing ultrasound screening. Orofacial clefts were present in 62 cases, an incidence of 1:624. The distribution of clefts was as follows: 18 (29\%) cleft lips, 25 (40\%) cleft lips with cleft palate, 17 (27\%) cleft palates only, one median cleft and one atypical cleft. Cleft lip with or without cleft palate was detected prenatally in 38/43 cases, a sensitivity of $88 \%$, but no case of cleft palate only was detected prenatally.

Offerdal et al. [6] evaluated prenatal detection by ultrasound on 101 fetuses with facial clefts in a large nonselected population (49,314 deliveries) and showed a significant statistical increase in detection rates (from 34 to $58 \%$ ) over the two 9-year periods. Isolated cleft palates amounted to $24 \%$, and none were detected prenatally.

Different 3D ultrasound techniques have been developed to image the fetal palate. Campbell et al. [7] were first to develop the "reverse face" view using 3D ultrasound. The fetal lips and alveolar ridge were examined in the frontal plane, and the face was then rotated $180^{\circ}$ on the vertical axis to examine the secondary palate. Eight cases of suspected orofacial clefting were examined. In one case with a left-sided cleft in the lips and the alveolar ridge, and with an intact hard palate, the correct diagnosis was made, but a cleft in the soft palate was missed.

Platt et al. [8] have described the "flipped face" view that is based on the multiplanar approach and which requires specific technical expertise. After volume acquisition, the image is rotated $90^{\circ}$ so that the cut plane is directed along a plane extending from chin to nose. The volume cut plane is then scrolled from chin to nose to examine sequentially the lower lip, mandible, and alveolar ridge; the tongue; the upper lip, maxilla, and alveolar ridge; and the hard and soft palates.

In the "angle insonation" technique developed by Pilu and Segata [9], in order to avoid acoustic shadowing from 
the alveolar ridge, the secondary palate is insonated at a $45^{\circ}$ angle in the sagittal plane, and 3D ultrasound is then used to reconstruct the axial and coronal planes. With this approach, the secondary palate was successfully visualized in 10 of 15 normal fetuses. In the fetus with CLP, the lesion in the secondary palate was clearly demonstrated, particularly in the coronal plane.

Tonni et al. [10] have demonstrated that 3D ultrasound enhanced the prenatal detection rate of CLP on an unselected low-risk pregnancy population of 1,856 at second trimester scan, with a detection rate of $100 \%$ in two cases of cleft lip and CLP.

The paper by Tonni et al. [10] underwent subsequent methodological analysis by Cunnigham [11] and achieved a $1 \mathrm{~A}$ evidence level. The statistical performance reported by Tonni et al. [10] has recently been confirmed by Bäumler et al. [12], who showed a sensitivity of $100 \%$ for detection of CLP in 81 high-risk fetuses, and a specificity of $90 \%$ using 2D mid-trimester ultrasound screening with axial $3 \mathrm{D}$ view.

The aim of this study is to describe a novel 3D volume and lightening techniques in the study of the fetal soft palate, with the uvula as the targeted anatomical landmark.

\section{Materials and methods}

The ultrasound examination was performed in a 25-weekold fetus using a Voluson E8 (General Electric Healthcare
Medical System, Milwaukee, WI) equipment with a transabdominal volumetric RAB4-8D probe. The patient was in her first uneventful pregnancy, and maternal body mass index (BMI) was $22\left(\mathrm{~kg} / \mathrm{m}^{2}\right)$. The fetal head was magnified until it occupied at least $75 \%$ of the screen, and two volume data sets were consecutively acquired at the time of fetal swallowing for online and further offline analysis. The time taken to acquire the volume of interest (VOI) was $6 \mathrm{~s}$, thus avoiding motion artifacts. The volumes were acquired with the function set to the surface mode and using a $20-40^{\circ}$ side-to-side sweep of the face with the optimized ultrasound settings as reported (quality high; second harmonic; gray map $=7$; speckle reduction $=0$ ). Volume contrast imaging with $3 \mathrm{~mm}$ slices was used to enhance contrast resolution. According to the "flipped face" technique and following acquisition of the volume, the tool bar (green bar) was extended from the chin to the nose enabling visualization of the VOI in multiplanar mode with 3D surface rendering in coronal plane of the hard, soft palate, and uvula (Fig. 1).

Antenatal ultrasound targeted single-shot-fast-spin-echo (SSSE) MR imaging was performed as a complementary diagnostic investigation at 30 weeks' gestation due to isolated, borderline ventriculomegaly $(12 \mathrm{~mm})$. Fetal MR imaging was conducted on a $1.5 \mathrm{~T}$ unit Intera Achieva ${ }^{\circledR}$, (Philips, Eindhoven, The Netherlands) using a sense body coil. T2-weighted images were acquired using a SSSE (repetition time of 15,000 ms; echo time: $120 \mathrm{~ms}$; echo train length: 140; acquisitions: 2 ; matrix: $139 \times 256$ ) in
Fig. 1 Volume of the fetal head was acquired with the function set to the surface mode and using a $\mathrm{B} 61^{\circ} / \mathrm{V} 50^{\circ}$ side-to-side sweep of the face with the optimized ultrasound settings as reported (quality high; second harmonic; gray map $=7$; speckle reduction $=0$ ). Volume contrast imaging with $3 \mathrm{~mm}$ slices was used to enhance contrast resolution. According to the "flipped face" technique, the tool bar (green bar) was extended from the chin to the nose enabling visualization of the volume of interest (VOI) in multiplanar mode with $3 \mathrm{D}$ surface rendering of the hard soft palate and uvula in coronal plane

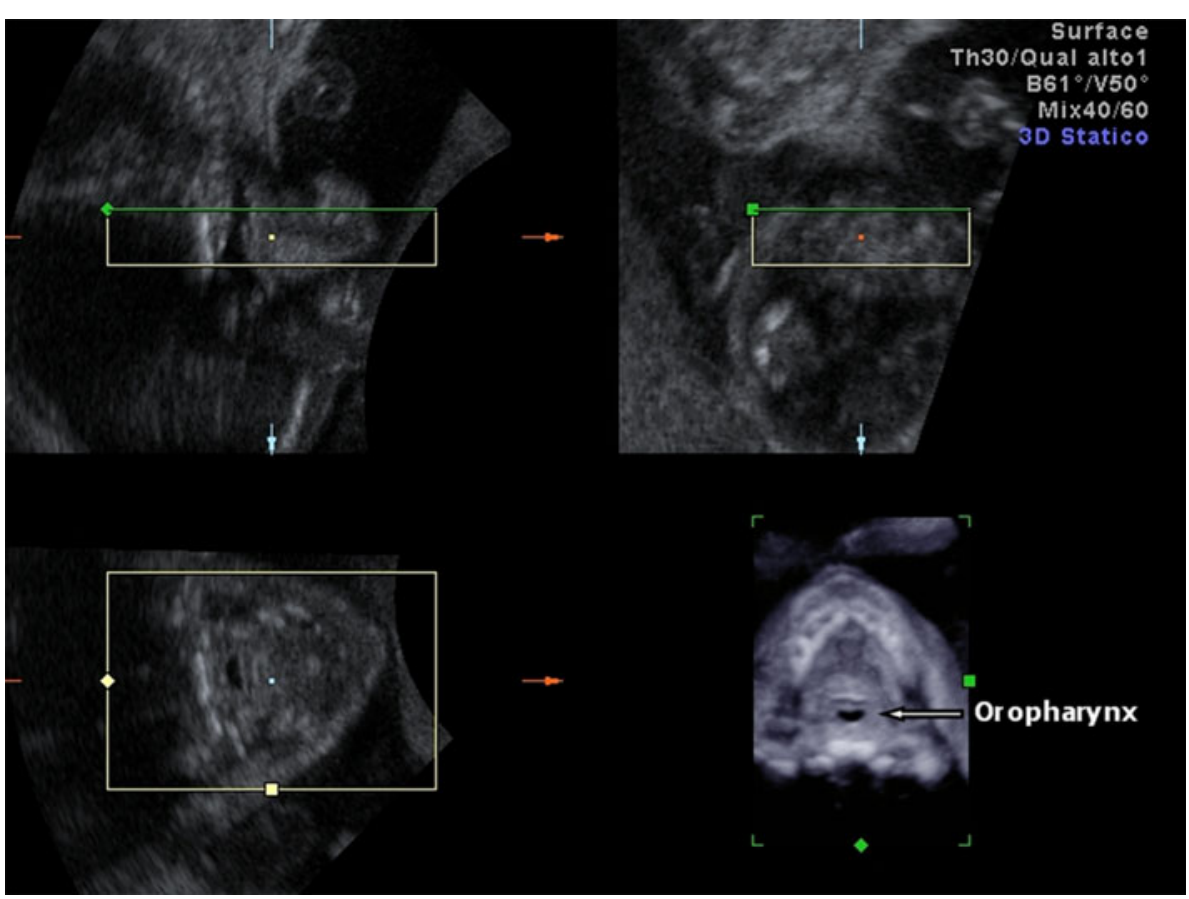


sagittal (Fig. 2) and coronal plane (Fig. 3). The hard and soft palates were clearly imaged as hyperintense signal.

Offline analysis of the 3D ultrasound volume data sets was performed on separate workstations with commercially available software (4D View, version 5.0; Luminary GE Medical Systems). 3D surface rendering was obtained in the coronal plane and then rotated and tilted to image the fetal uvula as the targeted anatomical landmark. The HDlive lightening system was applied at this time (Fig. 4a, b).

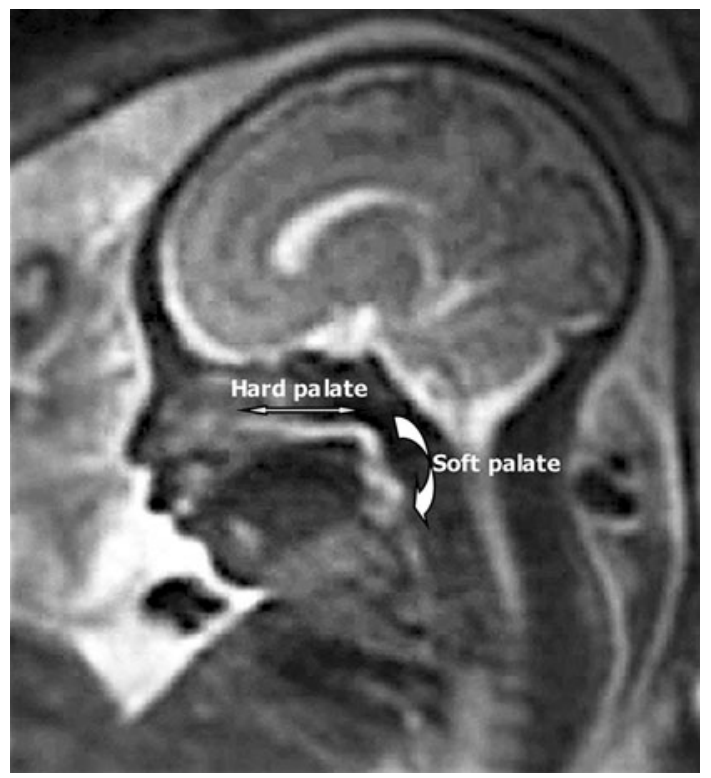

Fig. 2 Fetal MR imaging was conducted on a $1.5 \mathrm{~T}$ unit Intera Achieva ${ }^{\circledR}$, (Philips, Eindhoven, The Netherlands) using a sense body coil. T2-weighted images were acquired using a SSSE with a repetition time of 15,000 ms; echo time: $120 \mathrm{~ms}$; echo train length: 140; acquisitions: 2 ; matrix: $139 \times 256$. Note that the hard and the soft palate appeared as hyperintense signal in sagittal plane

\section{Discussion}

The antenatal detection rate of isolated cleft palate is low at $0-1.4 \%[3,6,13-16]$. Visualization of the soft palate is particularly challenging because of its curved anatomical arrangement, which results in shadowing by neighboring tissues [17] and difficult discrete recognition by ultrasound due to the lack of a landmark [18].

Faure et al. [19] examined 100 fetuses in a low-risk population at $17,22,27$, and 32 weeks' gestation to determine the normal 3D ultrasound view of the fetal palate at different gestational ages. The ultrasound scans were performed using the axial plane and an underside $3 \mathrm{D}$ view of the fetal palate. Also, the sonographic visualization rates of seven established anatomical landmarks of the fetal palate were computed for each gestational age.

The seven established anatomical landmarks of the fetal palate were identified in $42-100 \%$ of cases. Faure et al. [19] concluded that anterior axial 3D reconstruction of the fetal palate seen in underside view can provide diagnostic information on the integrity of the secondary palate and should be of value in diagnosing isolated secondary cleft palate or palatal involvement when cleft lip and alveolus are diagnosed. Faure et al. [20] studied 87 low-risk fetuses between 21 and 25 weeks' gestation to determine the normal 3D ultrasound view of the soft tissues of the fetal palate using a $30^{\circ}$-inclined axial view of the palate. The ultrasound images were then analyzed by two pediatric surgeons, who were able to assess the uvula and the velum in 80 and $90 \%$ of cases, respectively.

Using a median sagittal section in 2D ultrasound, Wilhelm and Borges [21] were able to visualize the uvula as an "equals sign" in $90.7 \%$ and the soft palate in $85.3 \%$ of 667 singleton pregnant women referred for detailed
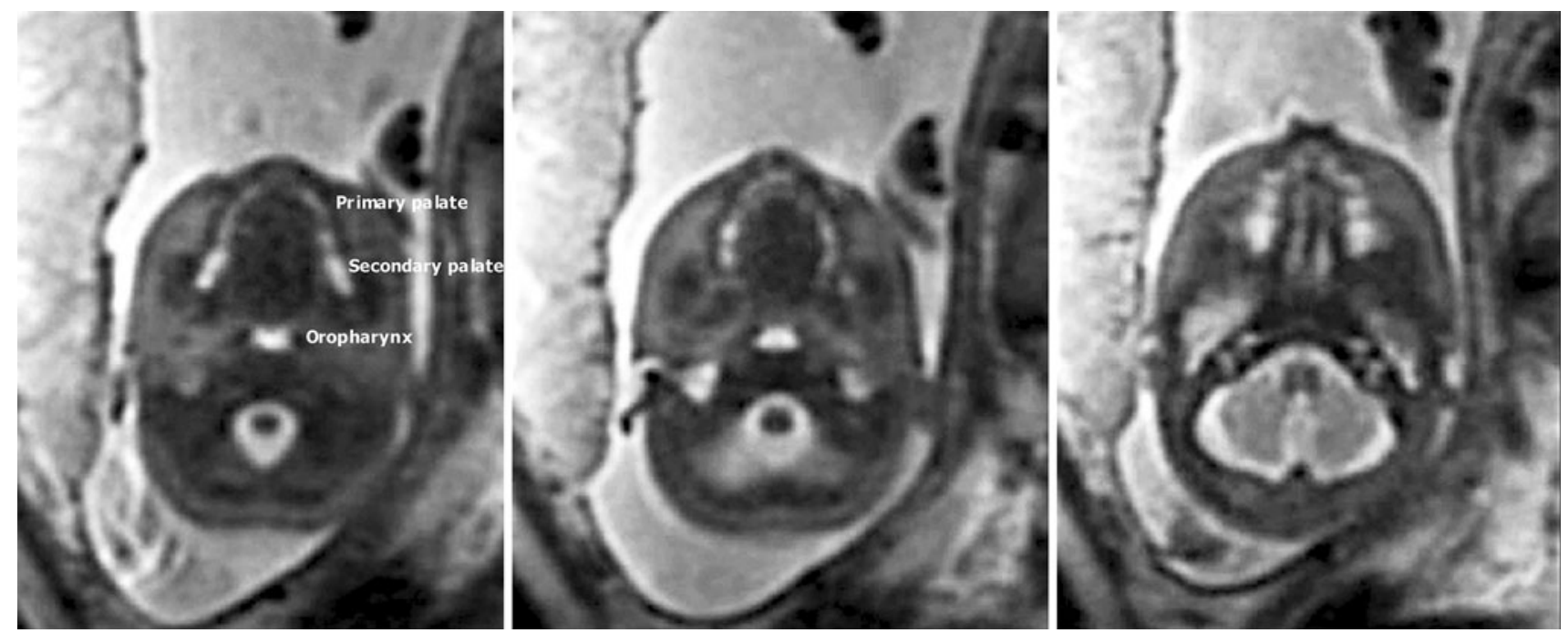

Fig. 3 Fetal MR imaging of the hard and soft palate appearing as hyperintense signal in coronal plane 
Fig. 4 Offline analysis of the 3D data sets was performed on separate workstations with commercially available software (4D View, version 5.0; Luminary GE Medical Systems). 3D surface rendering was obtained in the coronal plane and then rotated and tilted to image the fetal uvula as the targeted anatomical landmark. The HDlive lightening system was applied at this time $(\mathbf{a}, \mathbf{b})$
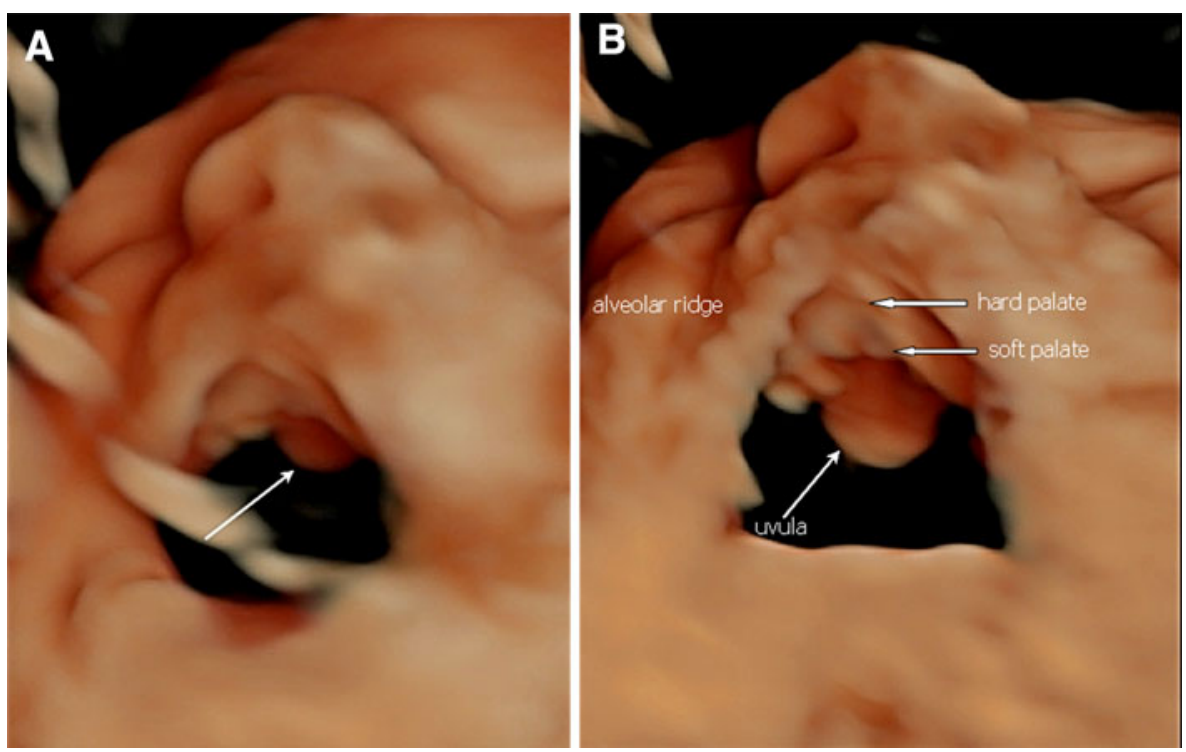

anomaly scan between 20 and 25 weeks of gestation. Visualization of at least one of the two structures (either the uvula or the soft palate) was successful in $98.4 \%$ of the cases. In one case, an isolated cleft palate (in an otherwise normal fetus) was diagnosed; in a case with a CLP, the cleft palate and the completely split uvula were detected. In $1.2 \%$ of cases, the examination did not provide sufficient information on either the uvula or the soft palate.

Wong et al. [23] examined the use of oblique planes from stored 3D ultrasound volumes of the fetal face for viewing the secondary palate in 5 [22] and 31 fetuses with confirmed normal secondary palates at different gestational ages (15-35 weeks). The secondary palate was viewed in three oblique planes targeted at the uvula: the oblique axial, the oblique sagittal and the reverse face view. The secondary palate could be viewed in the oblique axial and reverse face views in all 31 fetuses, and in all except two fetuses in the oblique sagittal view $<9$ weeks' gestation, at which age the uvula could not be identified. Wong et al. [23] concluded that the oblique axial, oblique sagittal and reverse face views targeted at the uvula enable the various aspects of the fetal secondary palate to be visualized on $3 \mathrm{D}$ ultrasound in the second and third trimesters of pregnancy. Moreover, the uvula could be used as a landmark for viewing the soft palate, but was not always easily identifiable before 19 weeks' gestation.

Martinez-Ten et al. [24] compared the performance of three sonographic techniques - the "reverse-face", "flipped-face" and "oblique-face" methods-for visualizing the hard and soft palate in diagnosing CLP in 60 fetuses at a gestational ages of 20-33 weeks. Out of the 10 fetuses with CLP, the defect involved the lip, alveolus and secondary palate in seven cases and the primary palate only in the remaining three. The upper lip and alveolar ridge were well visualized in all cases with all three methods. Involvement of the hard palate was diagnosed correctly in $71 \%(5 / 7)$ of the cases using the "reverse-face" view, in $86 \%(6 / 7)$ with the "flipped-face" view, and in $100 \%$ (7/7) with the "oblique-face" view. Involvement of the soft palate was diagnosed correctly in only one of the seven fetuses with defects of the secondary palate using the "flipped-face" and "oblique-face" views. However, curving the plane to follow the structure of the palate was key for achieving accurate anatomical reconstruction.

The soft palate lies at a $30^{\circ}$ angle with respect to the primary palate. Due to its curved anatomy, it is difficult to visualize it in its entirety using a single conventional orthogonal plane. In the axial plane, the undersurface of the soft palate close to the uvula is almost parallel to the scan plane and is obscured by the uvula. This is particularly awkward at a gestational age of $<22$ weeks, since the fetal secondary palate appears as a more or less straight structure in the sagittal plane, and the uvula can be identified only as a minuscule elevation from the palate. Over time, the uvula gradually elongates, and the soft palate becomes more identifiable, especially when the examination plane is rotated obliquely to avoid overlapping by the uvula. The secondary palate gradually increases in curvature (in both the sagittal and coronal planes) as the uvula lengthens with advancement of gestation. The uvula is a good landmark for directing the examination plane to the soft palate. The optimal time for visualizing the soft palate is after 20 weeks' gestation, probably at around 23-24 weeks, when the uvula can be identified consistently as a definite structure, and the palatal arch is present but not very marked. Starting from the three orthogonal planes, the ultrasound volume was scrolled, rotated or tilted to achieve the subsequent three oblique views while targeting the uvula [22]. 
The relative ease of viewing the uvula clearly, and hence the ease of visualization of the soft palate, was noted. We have reported a novel 3D technique based on surface rendering plus application of HDlive-a 3D lightening system with the ability to interrogate volumes with a light source and to help increase depth perception and reveal hidden details. It can also provide a deeper understanding of relational anatomy. Conventional ultrasound uses a fixed light source that reflects light off the surface of the skin. HDlive provides a movable virtual light source and calculates the pro-propagation of light through skin and tissue. The user can freely position the light at any angle relative to the ultrasound volume in order to illuminate areas of interest. Grisolia and Tonni [25] have very recently demonstrated that HDlive may be a useful 3D adjunct to second trimester fetal echocardiography and may help volume ultrasound achieve its best results ever. In conclusion, we have described a novel technique that can easily and rapidly be applied to capture snapshots of the hard and soft fetal palates, a finding that may be of critical importance in high-risk patients referred for level II ultrasound. The technique is best used after 20 weeks of gestation and during fetal swallowing, in accordance with the physiological development of the secondary palate. Maternal BMI and fetal positioning in utero are clinical variables that may interfere with reconstructed image quality. The time needed to acquire the volume is extremely short, so motion artifacts from the skull base are avoided. Prospective studies are needed to evaluate the feasibility of using this technical approach on the low-risk pregnancy population when operators and ultrasound facilities are locally available. As we reported, 3D rendering and lightening of the uvula may represent a complementary diagnostic armamentarium. Along with the 2D "equals sign", the technique may potentially contribute to aiding or enhancing prenatal ultrasound diagnosis of orofacial clefts, particularly those involving the secondary palate and the uvula.

Conflict of interest We Gabriele Tonni, Gianpaolo Grisolia declare not to have any financial relationship with the organization that sponsored the research. As authors we have had full control of all primary data and fully agree to allow the Journal to review their data if requested.

\section{References}

1. Coupland MA, Coupland AI (1988) Seasonality, incidence and sex distribution of cleft lip and palate births in Trent region (1973-82). Cleft Palate J 25:33-37

2. Cash C, Set P, Colemann N (2001) The accuracy of antenatal ultrasound in detection of facial clefts in a low risk screening population. Ultrasound Obstet Gynecol 18:432-436

3. Clementi M, Tenconi R, Bianchi F, Stoll C, Euroscan study group (2000) Evaluation of prenatal diagnosis of cleft lip with or without cleft palate by ultrasound: experience from 20 European registries. Prenat Diagn 20:870-875

4. Wang LM, Ky Leung, Tang M (2007) Prenatal evaluation of facial clefts by three-dimensional extended imaging. Prenat Diagn 27:722-729

5. Maarse W, Pistorius LR, Van Eeten WK, Breugem CC, Kon M, Van den Boogaard MJ, van Der Mink Molen AB (2011) Prenatal ultrasound screening for orofacial clefts. Ultrasound Obstet Gynecol 38:434-439

6. Offerdal K, Jebens N, Syvertsen T, Blaas HG, Johansen OJ, EikNes SH (2008) Prenatal ultrasound detection of facial clefts: a prospective study of 49,314 deliveries in a non-selected population in Norway. Ultrasound Obstet Gynecol 31:639-646

7. Campbell S, Lees C, Moscoso G, Hall P (2005) Ultrasound antenatal diagnosis of cleft palate by a new technique: the 3D "reverse face" view. Ultrasound Obstet Gynecol 25:12-18

8. Platt LD, Devore GR, Pretorius DH (2006) Improving cleft palate/cleft lip antenatal diagnosis by 3-dimensional sonography: the "flipped face" view. J Ultrasound Med 25:1423-1430

9. Pilu G, Segata M (2007) A novel technique for visualization of the normal and cleft fetal secondary palate: angled insonation and three-dimensional ultrasound. Ultrasound Obstet Gynecol 29:166-169

10. Tonni G, Centini G, Rosignoli L (2005) Prenatal screening for fetal face and clefting in a prospective study on low-risk population: can 3- and 4-dimensional ultrasound enhance visualization and detection rate? Oral Surg Oral Med Oral Pathol Oral Radiol Endod 100:420-426

11. Cunnigham ML (2006) Three-dimensional ultrasonography is superior to 2-dimensional ultrasonography in the detection of orofacial clefts during the second trimester of pregnancy. J Evid Based Dent Pract 6:278-279

12. Bäumler M, Faure JM, Bigorre M, Bäumler-Patris C, Boulot $P$, Demattei C, Captier G (2011) Accuracy of prenatal threedimensional ultrasound in the diagnosis of cleft hard palate when cleft lip is present. Ultrasound Obstet Gynecol 38:440-444

13. Grandjean H, Larroque D, Levi S (1999) The performance of routine ultrasonographic screening of pregnancies in the Eurofetus Study. Am J Obstet Gynecol 181:446-454

14. Shaikh D, Mercer NS, Sohan K, Kyle P, Soothill P (2001) Prenatal diagnosis of cleft lip and palate. Br J Plast Surg 54:288-289

15. Wayne C, Cook K, Sairam S, Hollis B, Thilaganathan B (2002) Sensitivity and accuracy of routine antenatal ultrasound screening for isolated facial clefts. Br J Radiol 75:584-589

16. Hanikeri M, Savundra J, Gillett D, Walters M, McBain W (2006) Antenatal transabdominal ultrasound detection of cleft lip and palate in Western Australia from 1996 to 2003. Cleft Palate Craniofac J 43:61-66

17. Campbell S (2007) Prenatal ultrasound examination of the secondary palate. Ultrasound Obstet Gynecol 29:124-127

18. Filly RA, Feldstein VA (2000) Ultrasound evaluation of normal fetalanatomy. In: Callen PW (ed) Ultrasonography in obstetrics and gynaecology. W.B. Saunders, Philadelphia, p 255

19. Faure JM, Captier G, Bäumler M, Boulot P (2007) Sonographic assessment of normal fetal palate using three-dimensional imaging: a new technique. Ultrasound Obstet Gynecol 29:159-165

20. Faure JM, Bäumler M, Boulot P, Bigorre M, Captier G (2008) Prenatal assessment of the normal fetal soft palate by threedimensional ultrasound examination: is there an objective technique? Ultrasound Obstet Gynecol 31:652-656

21. Wilhelm L, Borgers H (2010) The "equals sign": a novel marker in the diagnosis of fetal isolated cleft palate. Ultrasound Obstet Gynecol 36:439-444

22. Wong HS, Tait J, Pringle KC (2009) Examination of the secondary palate on stored 3D ultrasound volumes of the fetal face. Ultrasound Obstet Gynecol 33:407-411 
23. Wong HS, Tait J, Pringle KC (2008) The viewing of the soft and hard palate on routine 3D ultrasound sweep of the fetal facefeasibility study. Fetal Diagn Ther 24:146-154

24. Ten Martinez P, Pedregosa JP, Santacruz B, Adiego B, Barrón E, Sepúlveda W (2009) Three-dimensional ultrasound diagnosis of cleft palate: 'reverse face', 'flipped face' or 'oblique face'which method is best? Ultrasound Obstet Gynecol 33:399-406

25. Grisolia G, Tonni G (2013) Fetal echocardiography using HDlive. J Obstet Gynaecol Can 35:497 